\title{
Impact of Delay Spread on IEEE 802.15.4a Networks with Energy Detection Receivers
}

\author{
P. Medina* ${ }^{* 1}$ J. R. Gallardo ${ }^{2}$, J. Sánchez ${ }^{2}$, F. Ramírez-Mireles ${ }^{4}$ \\ ${ }^{1,3}$ Centro de Investigación Científica y de Educación Superior de Ensenada (CICESE) \\ Carretera Ensenada-Tijuana No. 3918, Zona Playitas, \\ Ensenada, B. C. México. C.P. 22860 \\ *pmedina@cicese.mx \\ ${ }^{2}$ Alcatel-Lucent University Ottawa \\ 600 March Road \\ Ottawa, Ontario, K2K 2E6 Canada \\ ${ }^{4}$ Instituto Tecnológico Autónomo de México (ITAM) \\ Río Hondo \#1, Col. Progreso Tizapán \\ Del. Álvaro Obregón C.P.01080, México, D.F.
}

\begin{abstract}
This work analyzes the impact of delay spread on IEEE 802.15.4a networks using energy detection (ED) receivers. Specifically, we review the typical values for delay spread in Ultra Wide Band (UWB) systems reported to date for indoor, outdoor and industrial environments, and study how the delay spread impacts the bit-error rate with and without Multiuser Interference (MUI).
\end{abstract}

Keywords: IEEE 802.15.4a, delay spread, energy detection (ED), impulse radio ultra wide band (IR-UWB), multiuser interference (MUI).

\section{RESUMEN}

En este trabajo se analiza el impacto del esparcimiento del retardo en redes IEEE 802.15.4a con receptor detector de energía (ED). Específicamente, se hace una revisión de los valores típicos del esparcimiento del retardo en las transmisiones de banda ultra ancha (UWB) reportados hasta la fecha en escenarios de interiores, exteriores e industriales, y además se estudia cómo el esparcimiento de retardo impacta la tasa de error de bit con y sin interferencia multiusuario (MUI).

\section{Introduction}

The IEEE 802.15.4 working group, whose goal is the standardization of low-rate and low-range wireless personal area networks (LDR-WPAN), has published a new physical layer option based on the TH-UWB (Time Hopping Ultra Wide Band) technology that intends to bring extra capabilities to this kind of networks [1]. The new physical layer is named IEEE 802.15.4a; its key novelties are higher data rates, ranging capabilities, and even more reduced energy consumption as compared to the previous physical layer options. All these characteristics considerably increase the number of applications and market opportunities of LDRWPANs.
A desirable feature in LDR-WPANs is that the transceiver devices should be low-cost; in this sense, the non-coherent reception with energy detection (ED) constitutes the most attractive reception scheme [3]. Keeping the low-cost idea in mind, the IEEE 802.15.4a modulation and coding schemes allow for the use of coherent and noncoherent reception, enabling networks of nodes with heterogeneous capabilities. Unfortunately, the non-coherent reception is more sensitive to the harsh conditions of the multipath UWB radio channel. 
Some experiments to characterize the UWB channel have already been carried out in different environments [4]-[12]. Among other interesting results, through these experiments it has been found that the delay spread is significantly high, as compared to the short duration of the transmitted pulses. In this work, the main contribution is on studying the degradation produced by the delay spread on the IR-UWB signal reception, specifically for the 802.15.4a-compliant devices using the ED reception technique. The paper starts describing the 802.15.4a modulation format in Section 2. Later, Section 3 summarizes the main results of some measurements on UWB signal propagation, its main focus being on the delay spread. Section 4 lists the negative consequences of the high delay spread on the IR-UWB signal reception. Finally, Section 5 concludes the document.

\section{IEEE 802.15.4a standard}

The original IEEE 802.15.4 specifications [2] are based on Direct Sequence Spread Spectrum (DSSS) technology using ISM bands on $868 \mathrm{MHz}$, $915 \mathrm{MHz}$, and $2.4 \mathrm{GHz}$, and reaching data rates of up to $250 \mathrm{kbps}$. The new IEEE 802.15.4a standard adds a new PHY layer based on impulsive UWB transmissions. This new option defines 16 Frequency Division Multiplexing (FDM) channels in the $0-10 \mathrm{GHz}$ range; 12 of these channels are 500 $\mathrm{MHz}$ wide (at $-3 \mathrm{~dB}$ bandwidth) and the 4 remaining channels are wider than $1 \mathrm{GHz}$. The standard does not restrict the pulse to any particular shape, but it is specified in terms of the correlation it must have with a reference pulse. For instance, for a $500 \mathrm{MHz}$ channel the reference signal is a root raised cosine pulse with roll-off parameter $\beta=0.6$ and 2 ns width.

The same pulse shapes can be used for the transmission of preamble symbols and for symbols included in the rest of a data frame; however, the modulation scheme used for the preamble is different from that used in data symbols. In what follows, we will describe only the modulation scheme used for symbols transmitted after the preamble, i.e. data symbols. For those interested in the preamble symbol format, detailed information can be found in the standard [1].
In the IR-UWB original idea, explained in [13], the time to transmit one bit is divided into multiple slots, and a single pulse is transmitted within each slot. The pulse position within each slot depends on the information bit and the TH sequence [14]. In the final IEEE 802.15.4a specification, the idea of transmitting several pulses per bit is maintained, nevertheless, the pulses are now transmitted one right after the other. This train of pulses is referred to as a burst. This approach can be seen as a clever way of varying the overall pulse width without the need to change the fundamental pulse shape or the bandwidth needed to transmit it. It also helps to reduce the spectral lines produced by PPM modulation [15]. The time needed to transmit one bit of information will be referred to as a symbol time $\left(T_{\text {dsym }}\right)$. The chip period $\left(T_{c}\right)$ is the portion of time necessary for a pulse transmission, and it constitutes the fundamental time unit in the standard (e.g. $T_{c}=2.0 \mathrm{~ns}$ for a $500 \mathrm{MHz}$ channel).

The chip period is inversely proportional to the Peak PRF (Pulse Repetition Frequency), defined as the maximum frequency at which pulses can be transmitted inside a burst. The standard also defines the Mean PRF as the number of pulses per burst divided by the duration of a data symbol. The Mean PRF is variable and it determines the data rate.

In the modulation scheme, named BPM-BPSK, the variation of the location or position of the burst within the symbol (BPM, Burst Position Modulation) is done simultaneously with the polarity or phase changes of its pulses (BPSK, Binary Phase Shift Keying). As an example, Fig. 1 sketches the data symbol structure for the mandatory data rate of $0.85 \mathrm{Mbps}$ at the Mean PRF of $15.60 \mathrm{MHz}$. The data symbol is $N_{c}$ chip periods long $\left(T_{\text {dsym }}=N_{c} T_{c}\right)$ and is divided into two BPM intervals $\left(T_{B P M}=T_{\text {dsym }} / 2\right)$. To reduce the intersymbol interference (ISI), the second half of each BPM interval is left unused as a way to insert a guard interval. The active part of a BPM interval is in turn subdivided into burst periods, each composed by $N_{c p b}$ chip periods $\left(T_{\text {burst }}=N_{c p b} T_{c}\right)$. As we mentioned previously, a pulse burst is a set of pulses consecutively transmitted at the Peak PRF 


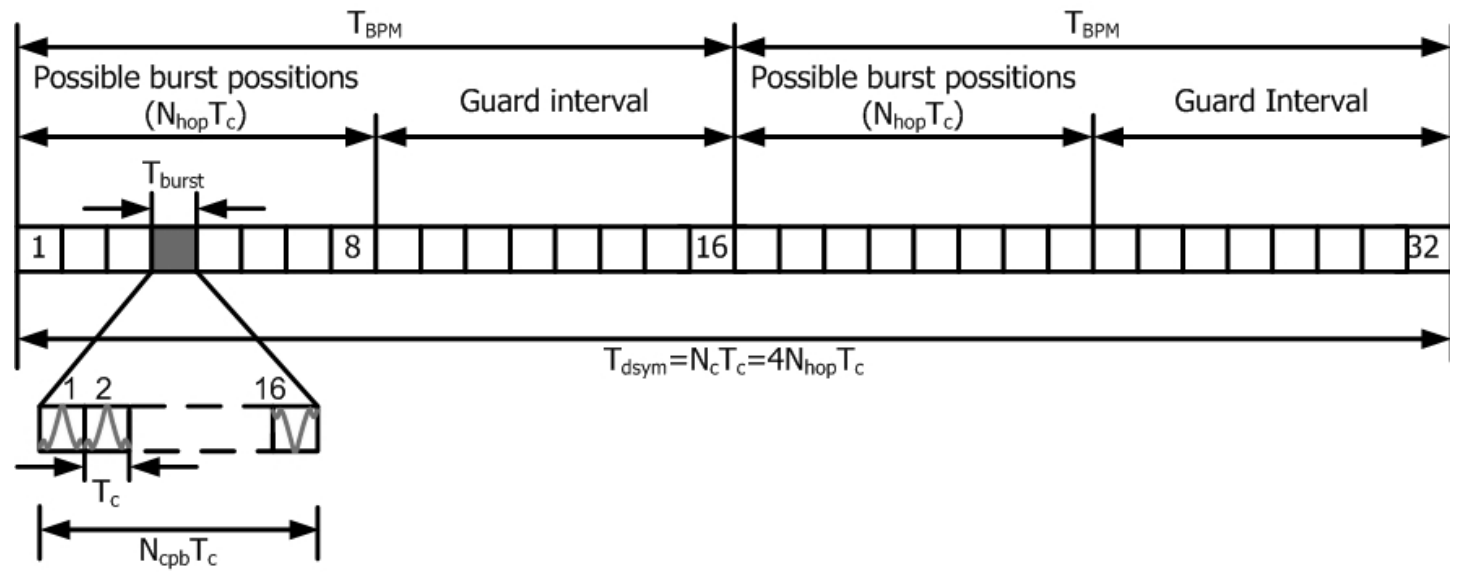

Figure 1. Example of a data symbol at the mandatory rate of 0.85 Mbps and Mean PRF of $15.60 \mathrm{MHz}$.

( $T_{c}$ seconds apart), and only one burst is transmitted within each symbol. The burst position within the symbol period is determined by two factors: 1) the information bit places the burst in one of the two BMP intervals; if the bit is " 0 " the burst has to be transmitted during one of the burst periods belonging to the first BPM interval, or, if the bit is "1", during the second BPM interval. 2) The burst position in the active portion of the BPM interval is set by the TH sequence, which pseudorandomly varies the burst position, thus reducing the multi-user interference (MUI) and improving the radiated signal spectrum shape.

The payload is protected through the use of two systematic channel coders: a Reed-Solomon coder (RS) and a convolutional coder, at rates 55/63 and
$1 / 2$, respectively. The $\mathrm{RS}$ redundancy bits are concatenated with the information bits and then introduced into the BPM modulator to be used as explained above. The convolutional redundancy bits are used by the BPSK modulator in such a way that the polarities of the different pulses within a burst are specified by them. All 802.15.4acomplaint devices must perform both kinds of encoding, but it is not mandatory to decode either of them.

Table 1 shows the operational parameters for the two mandatory Mean PRFs. The highlighted rows are the mandatory bit rate for each Mean PRF. The ingenious mixture between coding and modulation, combined with the Aloha access technique, enables the IEEE 802.15.4a PHY layer option to be used in heterogeneous networks [16].

\begin{tabular}{|c|c|c|c|c|c|}
\hline $\begin{array}{c}\text { Mean } \\
\text { PRF } \\
\text { (MHz) }\end{array}$ & $\begin{array}{c}\text { Chips } \\
\text { per } \\
\text { symbol }\end{array}$ & $\begin{array}{c}\text { Chips per } \\
\text { burst } \\
\left(\boldsymbol{N}_{\text {cpb }}\right)\end{array}$ & $\begin{array}{c}\text { RS } \\
\text { FEC } \\
\text { Rate }\end{array}$ & $\begin{array}{c}\text { Viterbi } \\
\text { Rate }\end{array}$ & $\begin{array}{c}\text { Bit } \\
\text { Rate } \\
\text { (Mbps) }\end{array}$ \\
\hline \multirow{3}{*}{$\begin{array}{c}15.60 \\
\text { (High) }\end{array}$} & 4096 & 128 & 0.87 & 0.5 & 0.11 \\
\cline { 2 - 6 } & 512 & 16 & 0.87 & 0.5 & 0.85 \\
\cline { 2 - 6 } & 64 & 2 & 0.87 & 0.5 & 6.81 \\
\hline \multirow{3}{*}{3.90} & 32 & 1 & 0.87 & 1 & 27.24 \\
\cline { 2 - 6 } (Low) & 512 & 34 & 0.87 & 0.5 & 0.11 \\
\cline { 2 - 6 } & 256 & 2 & 0.87 & 0.5 & 0.85 \\
\cline { 2 - 6 } & 128 & 1 & 0.87 & 0.5 & 1.70 \\
\hline
\end{tabular}

Table 1. Data rates, modulation and coding parameters of IEEE 802.15.4a physical layer. 


\section{UWB Channel delay spread}

This section summarizes the main published reports that include measurements that have been made with the goal of characterizing the UWB channel under different scenarios. The main focus is on results about delay spread $(\tau)$, its mean value $\left(\tau_{m}\right)$ and its root mean square value $\left(\tau_{r m s}\right)$; the latter is an estimation of the time interval where most of the received energy is concentrated. The measurements were made in different scenarios, using frequency- or time-domain techniques, and comprising several bandwidth sizes. The following subsections describe the scenarios and conditions under which the relevant measurements were made.

\subsection{Indoors}

One of the earliest measurements performed on the characterization of the UWB channel is reported in [4]. They were performed in a modern office building surrounded by large glass windows and including a laboratory area at the centre and small cubicles around it. The wall enclosing the laboratory is made of cement block, and the walls around the small offices are framed with metal rods and covered with plaster board. The transmit antenna was located in the laboratory, and the receiver was moved to each of the cubicles. Therefore, all of these measurements were made under non-line-of-sight (NLOS) conditions.

In [5] measurements are reported for several indoor environments: school, residential, and open industrial area. These measurements were truncated to $70 \mathrm{~ns}$ and the results combine measurements made under line-of-sight (LOS) and NLOS conditions. The measurements presented in [6] were also performed in indoor NLOS environments, inside a one-story building consisting of several densely furnished cubicle areas separated by drywall with vertical metal rods. The experiments in [7] were performed inside homes with different structure, age, size and clutter. Average results are presented independently for LOS and NLOS conditions.

All authors of the papers mentioned above conclude that the rms delay spread tends to increase as the distance between transmitter and receiver is increased. It also has been shown that the fading margin of UWB increases with distance [17]. They also agree that the delay spread is inversely proportional to the power value that sets the threshold under which impulse response samples are disregarded.

\subsection{Outdoors}

Regarding the outdoor environments, [8] describes the first measurement series in a wooded rural terrain. In [9] the measurements were performed in a forest environment and in a suburban area. Reference [10] includes measurements from office environments under LOS and NLOS conditions, however, these results can be classified as belonging to outdoor conditions since some correspond to signals generated in one of two adjacent buildings and measured at a point inside the empty space in between the buildings (LOS), while the others correspond to signals exchanged under similar conditions but the receiving point is blocked by a pillar and two glass doors (NLOS). The buildings consist of metal plated concrete walls with large windows and some trees around them.

\subsection{Industrial}

Measurement campaigns have been scarcer in industrial environments than in any other type of environment. In [11] and [12] the authors performed impulse response measurements in an incinerator hall. The hall consisted mainly of corrugated iron walls and was equipped with a large reaction chamber, some pumps, pipes, and tanks. Their results show that there is not a noticeable difference between the power profile of LOS and short-distance (less than $8 \mathrm{~m}$ ) NLOS.

\subsection{Summary of delay spread values}

Table 2 summarizes the results of the measurements mentioned above. Data not provided at the sources was left blank in the corresponding table entry. In the data shown, we can observe that the reported values have a broad range even in similar scenarios. The reason for that is the well known dependence of the channel impulse response on the considered bandwidth and the distance between transmitter and receiver [18]. 
The indoor scenarios generally have a relatively dense delay spread. However, they show the smallest rms values, which indicates that most of the energy concentrates at the beginning of the impulse response. This effect has been extensively pointed out in [19], where it is explained that even though the distances between transmitter, receiver and scatterers are short in these environments, the effect of the high resolution provided by the wide spectrum of UWB systems makes the number of scatters to be relatively large, which allows the system to discern among a larger number of strong individual components arriving at the beginning of the impulse response than what could be detected by a narrow band system in the same scenario. The outdoor scenarios show the smallest delay spread, but their higher rms values reveal that the energy is distributed more evenly over the observed time interval; in other words, outdoor scenarios are more time-dispersive. The industrial scenario shown has a relatively high delay spread and the energy is also evenly distributed. In general, it can be observed that the NLOS condition tends to increase the energy spread, as compared to the LOS condition in a similar environment, being the outdoor scenarios the ones where this difference is more accentuated, and the industrial scenario where this difference is less significant. It has been shown that the channel capacity for NLOS with larger delay spread is lower than the channel capacity for LOS with shorter delay spread [20].

\begin{tabular}{|c|c|c|c|c|c|c|}
\hline Ref. & Scenario & $\begin{array}{c}\tau \\
\text { (ns) }\end{array}$ & $\begin{array}{c}\tau_{m} \\
\text { (ns) }\end{array}$ & $\begin{array}{l}\tau_{r m s} \\
\text { (ns) }\end{array}$ & $\begin{array}{l}\text { Band- } \\
\text { width } \\
\text { (GHz) }\end{array}$ & $\begin{array}{l}\text { Distance } \\
\text { (m) }\end{array}$ \\
\hline \multicolumn{7}{|c|}{ Indoors } \\
\hline [4] & $\begin{array}{l}\text { NLOS } \\
\text { Office }\end{array}$ & 150 & - & - & $>1$ & $<17$ \\
\hline [5] & $\begin{array}{l}\mathrm{N}) \mathrm{LOS} \\
\text { Various }\end{array}$ & $40-70$ & - & $\begin{array}{l}8.60- \\
18.60\end{array}$ & - & variable \\
\hline [6] & $\begin{array}{l}\text { NLOS } \\
\text { Office }\end{array}$ & 150 & - & 15 & $1.25-2.75$ & 10 \\
\hline \multirow{2}{*}{ [7] } & $\begin{array}{c}\text { LOS } \\
\text { Residential }\end{array}$ & \multirow{2}{*}{70} & - & 4.7 & \multirow{2}{*}{$\begin{array}{l}4.375- \\
5.625\end{array}$} & \multirow{2}{*}{$1-10$} \\
\hline & $\begin{array}{c}\text { NLOS } \\
\text { Residential }\end{array}$ & & - & 8.2 & & \\
\hline \multicolumn{7}{|c|}{ Outdoors } \\
\hline \multirow{2}{*}{ [8] } & $\begin{array}{l}\text { LOS } \\
\text { Forest }\end{array}$ & \multirow{2}{*}{$\begin{array}{c}< \\
100\end{array}$} & 13.77 & 31.02 & \multirow{2}{*}{1.3} & 3 \\
\hline & $\begin{array}{l}\text { NLOS } \\
\text { Forest }\end{array}$ & & 22.49 & 38.08 & & 10 \\
\hline \multirow{2}{*}{ [9] } & $\begin{array}{l}\text { NLOS } \\
\text { Forest }\end{array}$ & - & $1-4.5$ & $5-11$ & \multirow{2}{*}{$3-6$} & $10-60$ \\
\hline & $\begin{array}{l}\text { (N)LOS } \\
\text { Suburban }\end{array}$ & - & $2-17$ & $7-32$ & & $8-15$ \\
\hline \multirow{2}{*}{ [10] } & $\begin{array}{l}\text { LOS } \\
\text { Office }\end{array}$ & \multirow[b]{2}{*}{ - } & 24.1 & 55.1 & \multirow{2}{*}{$3-6$} & $3-24$ \\
\hline & $\begin{array}{l}\text { NLOS } \\
\text { Office }\end{array}$ & & 83.5 & 97.8 & & $8-15$ \\
\hline \multicolumn{7}{|c|}{ Industrial } \\
\hline \multirow{2}{*}{$\begin{array}{l}{[11]} \\
{[12]}\end{array}$} & $(\mathrm{N}) \mathrm{LOS}$ & \multirow{2}{*}{160} & - & 30 & \multirow{2}{*}{$3.1-10.6$} & 2 and 4 \\
\hline & NLOS & & - & 40 & & 8 \\
\hline
\end{tabular}

Table 2. Summary of delay spread results 
Before concluding this section, it is important to emphasize that we are aware that it is still necessary to perform more measurements in order to characterize the UWB channel in an extensive way [21]. We also need to keep in mind that these measurements correspond not only to different environments, but also to different pulse shapes and frequency bands. Rather than making precipitated conclusions about the general behavior of the UWB channel, the purpose of this section is simply to show the delay spread typical values in different scenarios.

Reference [21] describes specific models for the different scenarios mentioned above. These models are generally accepted and commonly used in the literature. The model known as CM1 corresponds to a residential environment with LOS. The model referred to as CM2 corresponds to a residential environment with NLOS. We will use these models in the following section, which shows numerical results relating the delay spread with the corresponding bit error rate (BER).

\section{Impact of delay spread on ED receiver performance}

Fig. 2 sketches a block diagram of the energy detection [3], which consists of a pass-band filter, a square law device, an integrator, and a sampling device. In this way the output at the energy detector are the samples of the energy gathered in the band of interest during the integration or sampling time $T$.

According to this block diagram the sample $v(n T)$ will be given by the following expression:

$$
v(n T)=\frac{1}{T} \int_{(n-1) T}^{n T} y^{2}(t) d t
$$

In what follows, we show, through simulations, the negative effects that the delay spread has on BER performance of an IEEE 802.15.4a compliant device using the ED receiver. In all the results, the $0.85 \mathrm{Mbps}$ mandatory data rate was used. As shown in Table 1, this rate can be achieved with 4 or 16 pulses per burst corresponding to the 3.9 and 15.6 Mean PRFs, respectively. In the ED detector, an integration time of either $2 \mathrm{~ns}$ or $8 \mathrm{~ns}$ is used, corresponding to the duration of one and four pulses.

In order to evaluate the ED receiver performance, a simulator in Matlab based on the one developed by [22] was implemented. In the simulator scenario, there are one or more users generating independent packets following a Poisson process and trying to make them reach a receptor node. Originally, the receiver node is always executing a synchronization process, once synchronized to the transmitter, it performs a coarse channel estimation, looks for the start of the frame, and then proceeds to the data symbols decoding. Even though we always assume that the receiver is perfectly synchronized to the transmitter of interest, we still keep the coarse channel estimation because it is needed in the data symbol decoding. The coarse channel estimation involves the averaging of various preamble pulses to provide a coarse pulse energy spread profile. At the receiver, two decision variables are created for the decoding process $(\mathrm{HO}$ and $\mathrm{H} 1)$; these variables are the result of the addition of the corresponding energy channel samples, considering that there was a burst transmission in the burst position corresponding to either ' 0 ' or ' 1 ' bit, respectively. The estimated energy profile takes into account when the samples are picked for each addition.

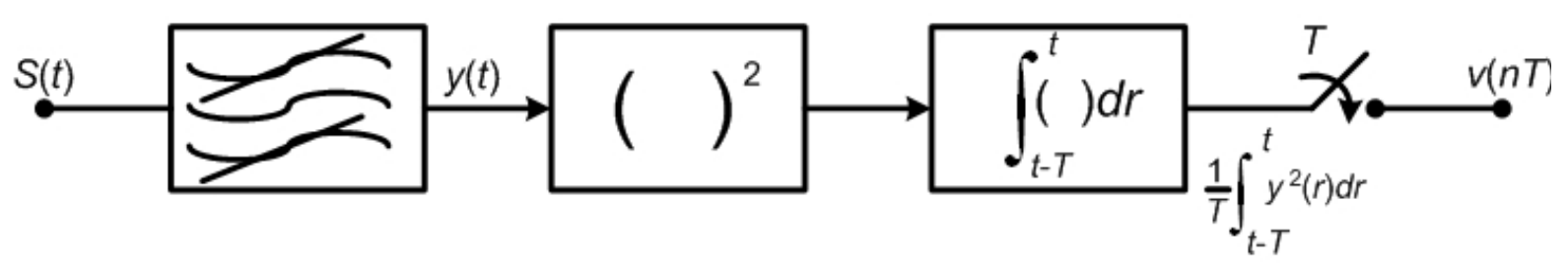

Figure 2. Block diagram of Energy Detector. 
Fig. 3 shows the BER performance of the ED receiver considering just one user without interference, in both we used 4 pulses per burst and an integration time of $8 \mathrm{~ns}$. It can be observed that $\mathrm{CM} 2$ performance is slightly worse compared to $\mathrm{CM} 1$, this is due to a bigger rms delay spread in CM2 compared to CM1, as can be seen in Table 2. That means that, in CM2 more noise energy is introduced into the decision variables and this increases the error probability. The degradation in both channel models with respect to the nomultipath channel results evident, this is also consequence of the delay spread.

Theoretically, the BER performance under dispersive channels could be closer to the nomultipath channel if during the creation of the decision variables something more elaborated could be done than simply adding the energy samples. For example in [23] and [24], the energy samples are weighted before the addition process. The weighting is based in the estimated energy profile, giving a high weight to the samples where signal energy is big compared to noise energy, and a low weight to the samples containing too much noise energy. This approach, however, requires more accurate channel estimation and could require very short integration times.

Even with the coarse channel estimation, there still are two options that could enable a better performance in the energy detector. Those are the shortening of the integration time and the increase of the number of pulses per burst. Fig. 4 shows what happens to BER performance when the integration time is reduced and/or the number of pulses per burst is increased in the CM1. It is observed that in the case of 4 pulses per burst, the reduction of the integration time from $8 \mathrm{~ns}$ to $2 \mathrm{~ns}$ brings a noteworthy improvement on performance. The increasing of the burst length from 4 to 16 pulses brings improvement as well, however, this advantage decreases as the SNR increases; this does not happen for the case of the integration time reduction, where the advantage persists even for bigger SNRs.

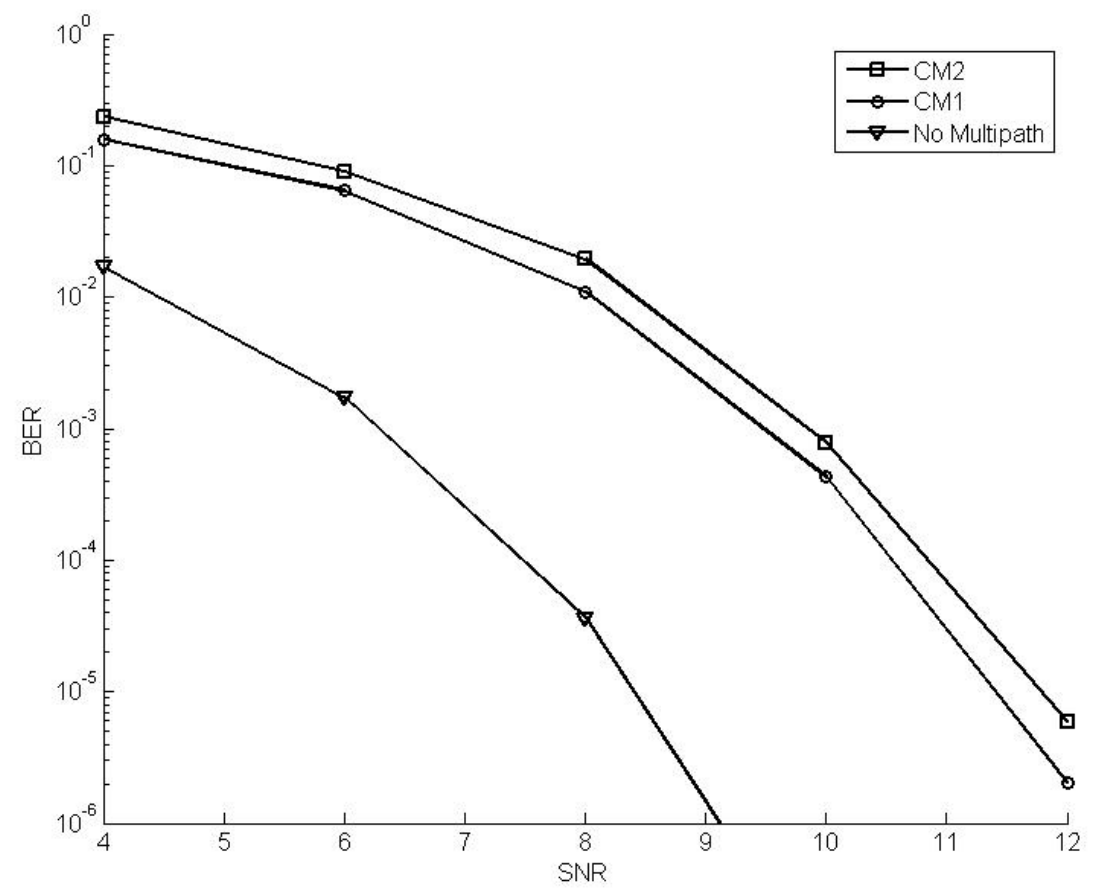

Figure 3. BER performance considering an ED receiver, only one user perfectly synchronized, CM1 and CM2 multipath channels without interference. 


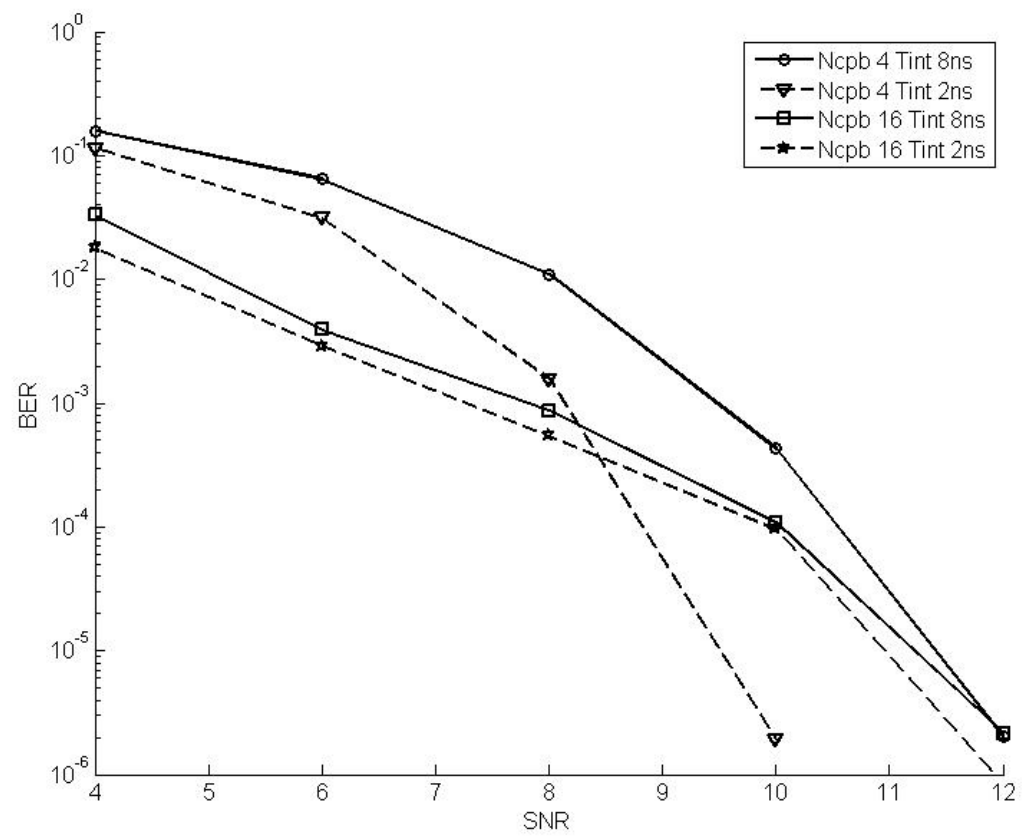

Figure 4. BER tendency for a perfectly synchronized user using an ED receiver under the multipath channel CM1, changing the number of chips per burst $\left(N_{c p b}\right)$ and the integration time $\left(T_{i n t}\right)$.

Fig. 4 suggests that a deeper analysis could be very useful if we are interested in adapting the physical layer parameters to the more appropriated choice of the link conditions. For example, energy could be saved at the transmitter if fewer pulses per burst are used and still achieve a satisfactory BER; or resources could be saved at the receiver using the shortest possible integration time that meet a satisfactory performance.

There are two intriguing results on Fig. 4: 1) when the burst length is large, reducing the integration time does not bring notorious improvements on performance. 2) The combination of more pulses per burst and shorter integration time does not seem to be the best choice after a SNR threshold. This SNR threshold reduces for smaller integration times. Fig. 4 suggests that the integration time should match the duration of the pulse burst. More specifically, for the $N_{c p b}=4$ case, a larger integration time $T_{\text {int }}=8 \mathrm{~ns}$ allows more noise to enter in the decision variable, and a shorter $T_{\text {int }}=$ 2 ns allows to integrate over the period where the received signal is present. For a reduced $T_{\text {int }}$, there is less noise but no less signal energy. For the $N_{c p b}$ $=16$, there is no gain in going from $T_{\text {int }}=8 \mathrm{~ns}$ to $T_{\text {int }}$ $=2 \mathrm{~ns}$ since both integration times cover the presence of signal. For a reduced $T_{\text {int }}$, there is less noise but also less signal energy.

To this point, we have considered a unique transmitting user, now we show in Fig. 5 what happens when an interfering user is introduced. In this case, we have again supposed that the receiver is perfectly synchronized to the transmitter of interest, and there is an unsynchronized interfering transmitter that transmits simultaneously, in such a way that each data symbol of the transmitter of interest contains a randomly located interfering burst. For comparison purposes, Fig. 5 also contains the no-interference case results.

It is observed in Fig. 5 that, for low SNR levels, the interference is not distinguished from noise. After an SNR level, the interference clearly exceeds noise, until there is a point where an SNR increase does not improve significantly the BER. Obviously, 


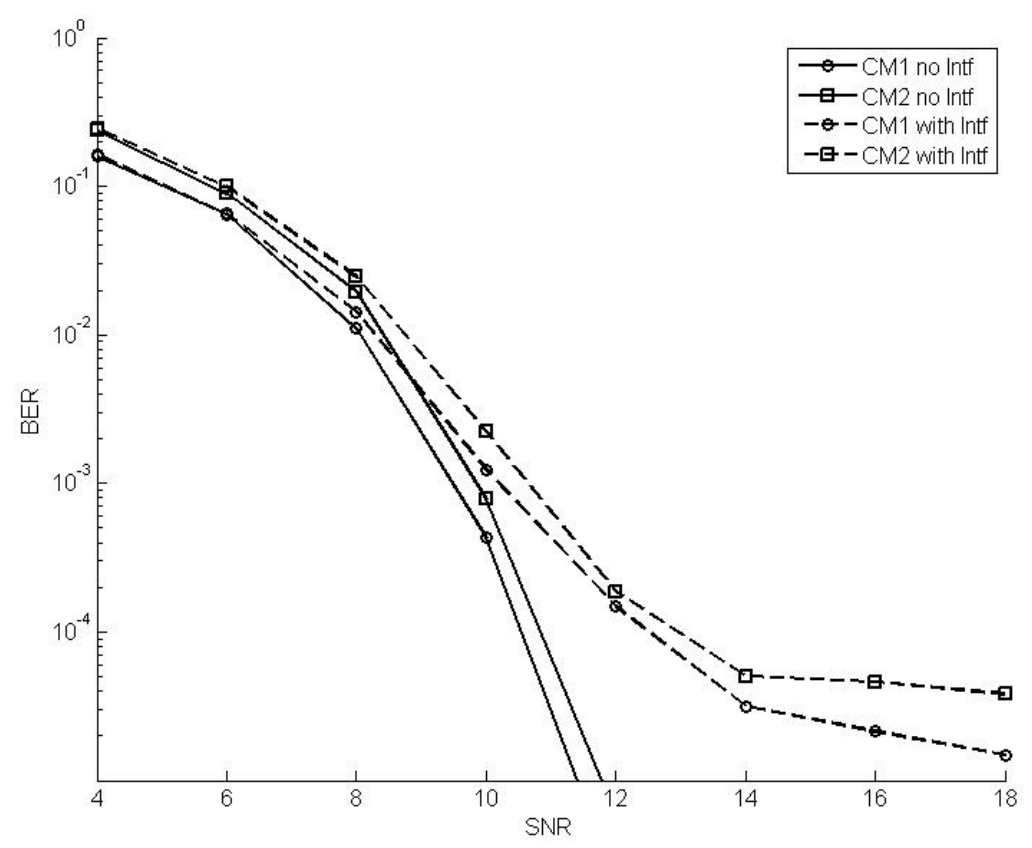

Figure 5. BER tendency for one perfectly synchronized transmitter, and one interfering node received at the half of the power of the transmitter of interest.

this point depends on the ratio of the powers of the transmitter of interest and the interfering transmitter. Again, this behavior has been observed for coherent receivers [25] but we think it deserves a deeper analysis if a more adequate parameter selection is required. For example, if we are interested in saving energy, unnecessary energy wastes could be avoided by adjusting the transmission power to the minimum necessary level that reaches the lower possible BER under interference conditions because, as can be seen in Fig. 5, the extra energy is unnecessarily wasted [26].

Finally, it is important to underline that the delay spread has a very significant impact on the performance because it causes to extend the time interval during which non-negligible signal replicas arrive, which means that the intervals during which burst collisions can occur are also enlarged as a consequence. The end result is a larger probability of collision, and hence, a stronger degradation due to MUI than what would be observed if the delay spread were not present occurs.

\section{Conclusions}

This work reviews the main measurement results that are currently available for the UWB channel characterization, with special emphasis on the delay spread. In addition to that, it also shows through simulations the degradation that the delay spread has on the performance of an ED receiver compatible with the IEEE 802.15.4a standard. To be more specific, it was found that, because of the inclination of ED receivers to increase the amount of detected noise energy as the rms delay spread increases, the performance observed in channels with larger delay spread was worse than that related to less dispersive channels. It was found as well that the delay spread worsens the problem of multi-user interference because it increases the burst collision probability.

There are solutions to these problems that may still allow for the ED receptor, which is very convenient for use in these types of networks for its simplicity and low cost. The simplest standard compatible solutions aim to increase the number of pulses per burst and/or reducing the ED integration time. It has 
been observed from the results that an appropriated choice of the numbers of pulses per burst and the integration time can help the ED receiver on achieving a better performance; however, this choice is not as direct as it seems, again, because of the delay spread. The main focus of our next research activity is to find the adequate rules for this transmission-reception parameter selection.

\section{References}

[1] IEEE 802.15.4a-2007. Wireless Medium Access Control (MAC) and Physical Layer (PHY) Specifications for Low-Rate Wireless Personal Area Networks (LRWPANs). Amendment 1: Add alternate PHYs. IEEE Standard 802.15.4a, 2007 Edition.

[2] IEEE 802.15.4-2006. Wireless Medium Access Control (MAC) and Physical Layer (PHY) Specifications for Low-Rate Wireless Personal Area Networks (LRWPANs). IEEE Standard 802.15.4, 2006 Edition.

[3] Urkowitz $H$. , Energy detection of unknown deterministic signals, Proc. of IEEE, vol. 55, April 1967, pp. 523-531.

[4] Win M. Z., Scholtz R. A. \& Barnes M. A., Ultra-wide bandwidth signal propagation for indoor wireless communications, IEEE International Conference on Communications 1997.

[5] Hall D. J., Indoor Propagation Analysis Techniques for Characterization of Ultra-wideband RF Environments, Wireless personal communications: bluetooth tutorial and other technologies, Kluwer Academic Publisers, 2002.

[6] Yano S., Investigating the ultra-wideband indoor wireless channel, IEEE 55th Vehicular Technology Conference, 2002.

[7] Ghassemzadeh S.S., Jana R., Rice C.W., Turin W. \& Tarokh V., A statistical path loss model for in-home UWB channels, IEEE Conference on Ultra Wideband Systems and Technologies, 2002.

[8] Win M. Z., Ramírez-Mireles F., Scholtz R. A. \& Barnes M. A., Ultra-wide bandwidth signal propagation for outdoor wireless communications, IEEE 47th Vehicular Technology Conference, 1997, vol. 1, pp. 251-255.
[9] Di Renzo M., Graziosi F., Minutolo R., Montanari M. \& F. Santucci, The ultra-wide bandwidth outdoor channel: From measurement campaign to statistical modelling, Mobile Networks and Applications, Volume 11, Number 4, August 2006 , pp. 451-467(17).

[10] Kim C.W., Sun X., Chiam L.C., Kannan B., Chin F.P.S. \& Garg H.K., Characterization of ultra-wideband channels for outdoor office environment, IEEE Wireless Communications and Networking Conference, 2005.

[11] Karedal J., Wyne S., Almers P., Tufvesson F. \& Molisch A. F., UWB channel measurements in an industrial environment, IEEE Global Telecommunications Conference, 2004.

[12] Karedal J., Wyne S., Almers P., Tufvesson F. \& Molisch A. F., Statistical analysis of the UWB channel in an industrial environment, IEEE 60th Vehicular Technology Conference, 2004.

[13] Scholtz R. A., Multiple Access with Time-Hopping Impulse Modulation, invited paper, Proceedings of IEEE MILCOM conference, December 1993.

[14] Ramírez-Mireles F., Impulse Radio Ultrawideband: IR-UWB signal design and system performance, ISBN 978-3-639-26607-8, published by VDM Verlag, Germany, 2010.

[15] Ramírez-Mireles F., Performance of UWB NOrthogonal PPM in AWGN and Multipath Channels, in IEEE Transactions on Vehicular Technology, Vol. 56, No. 3, May 2007, pp. 1272-1285.

[16] Molish F. A., Orlik P., Sahinoglu Z. \& Zhang J., UWB-based sensor networks and the IEEE 802.15.4a standard - a tutorial, First International Conference on Communications and Networking in China, 2006.

[17] Bastidas-Puga E.R., Ramírez-Mireles F., MuñozRodríguez D., On Fading Margin in Ultrawideband Communications over Multipath Channels, IEEE Transactions on Broadcasting, Vol. 51, Issue 3, September 2005, pp. 366-370.

[18] Hashemi H., Impulse Response Modeling of Indoor Radio Propagation Channel, IEEE Journal on Selected Areas in Communications, 1993.

[19] Kunisch J. \& Pamp J., Measurement results and modeling aspects for the UWB radio channel, IEEE Conference on Ultra Wideband Systems and Technologies, 2002. 
[20] Ramírez-Mireles F., On the Capacity of UWB over Multipath Channels, IEEE Communications Letters, Vol. 9, Issue 6, June 2005, pp. 523-525.

[21] Molish F. A., Balakrishnan K., Cassioli D., Chong C., Emami S., Fort A., Karedal J., Kunisch J., Schantz H., Schuster U. \& Siwiak K., IEEE 802.15.4a channel model - final report, 2004.

[22] Flury M., Merz R., Le Boudec J.-Y. \& Zory J., Performance Evaluation of an IEEE 802.15.4a Physical Layer with Energy Detection and Multi-User Interference, IEEE International Conference on Ultra-Wideband (ICUWB 2007), September 2007.

[23] D'amico A. A., Megali U., Arias-de-Reyna E., Energy Detection UWB Receivers with Multiple Energy Measurements, IEEE Transactions on Wireles Communications, vol. 6, no. 7, June 2007.

[24] Tian Z., Sadler B.M., Weighted energy detection of ultra-wideband signals, IEEE 6th Workshop on Signal Processing Advances in Wireless Communications. New York, USA, June 2005.

[25] Ramírez-Mireles F., Quantifying the Degradation of Combined MUI and Multipath Effects in Impulse-Radio UWB, in IEEE Transactions on Wireless Communications, Vol. 6, No. 8, August 2007, pp. 28312836.

[26]. Ramírez-Mireles F., Transmission Power Management for IR-UWB WSN Based on Node Population Density, in "Wireless Sensor and Actor Networks II", IFIP International Federation for Information Processing Book Series, ISBN 978-0-387-09440-3, published by Springer Boston, May 2008, pp. 37-48. 


\section{Paul MEDINA}

He received the B.Eng. degree from Instituto de Tecnología de Sonora, Obregon, Mexico, and the M.Sc. degree from Centro de Investigación Científica y de Educación Superior de Ensenada (CICESE), Ensenada, Mexico, both in electrical engineering. From July to September 2005, he worked as a research associate at the Broadband Wireless and Internetworking Research Laboratory of the University of Ottawa, Canada. From October 2005 to January 2007, he worked as a lead engineer in projects related to routing and access control in wireless sensor networks as well as IP telephony over wireless LANs. From August 2007 to July 2008, he was a visiting student at the University of Western Ontario, Canada. Currently, he is a Ph.D. student at CICESE. His main interest areas of study are related to wireless access networks.

\section{Jose R. GALLARDO}

He received the D.Sc. degree in EE from the George Washington University, Washington, DC, in 2000. He was a professor of telecommunications at Centro de Investigación Científica y de Educación Superior de Ensenada (CICESE) in Ensenada, Mexico, from 2000 to 2008. From August 2007 to July 2008, he was a visiting professor at the University of Western Ontario, Canada. He also spent almost two years, from August 2008 to May 2010, as a visiting professor at the University of Ottawa, Canada. He is currently with the Alcatel-Lucent University, located in Ottawa, Canada. His main areas of interest are quality of service, call admission control, service differentiation, medium access control and routing protocols, as well as network modeling, analysis and simulation with emphasis on wireless local-area networks and wireless sensor networks.

\section{Jaime SANCHEZ}

He Received an engineering degree in communications and electronics from Instituto Politécnico Nacional (IPN), Mexico City (1976); an M.Sc. degree in electronics and telecommunications from CICESE, Ensenada, Mexico (1979); and a D.Sc. degree in electrical engineering, major in communications, from The George Washington University (2001). Since 1979, he has a research and faculty position at the Electronics and Telecommunications Department at CICESE, Ensenada B.C., Mexico. Dr. Sanchez was a visiting scholar at the University of Arizona Tucson (ECE) in 1997, and a visiting scholar at The University of Texas at Austin (ECE) in 2008. He has published several papers in IEEE Journals and International Conferences. His current research interests include wireless networks, software radio, channel modeling, multicarrier modulation (OFDM), and MIMO-OFDM. He is an IEEE member. 


\section{Fernando RAMIREZ-MIRELES}

He received his Ph.D. in E.E. from the University of Southern California (USC), Los Angeles, CA, USA. He has over 5 years of industrial experience in companies in the Silicon Valley and over 10 years of experience in academia. Presently, he is a full professor at Instituto Tecnológico Autónomo de México (ITAM), in Mexico City. He has authored or co-authored over 30 technical articles and book chapters in areas including modulation and signal design for UWB communications, spread spectrum multiple access performance, performance in multipath channels, effects of crosstalk in DSL, and speech recognition. His work on UWB is frequently cited. He holds 10 international patents on DSL with one more pending. He was a coordinator at the 1st and 2nd French- Mexican Summer Schools in Telecommunications. He acts as a reviewer for professional publications and conferences. He was a Fulbright Scholar while studying his Ph.D. program at USC. He is listed in International Who's Who of Professionals. He is a member of Tau Beta Pi and the Society of Hispanic Professional Engineers. He can be reached at ramirezm@ieee.org. 